Tighlighted Paper selected by Oditor-in-Ghief

\title{
Characterization of Fatty Acid Profile in the Liver of SHR/NDmcr-cp (cp/cp) Rats, a Model of the Metabolic Syndrome
}

\author{
Shizuyo Tanaka, ${ }^{a}$ Yurie Yagi, ${ }^{a}{ }^{\dagger}$ Tohru Yamazaki, ${ }^{a}$ Atsushi Mitsumoto, ${ }^{b}$ Daisuke Kobayashi, ${ }^{a}$ \\ Naomi Kudo, ${ }^{a}$ and Yoichi Kawashima*,a \\ ${ }^{a}$ Faculty of Pharmaceutical Sciences, Josai University; 1-1 Keyakidai, Sakado, Saitama 350-0295, Japan: and \\ ${ }^{b}$ Faculty of Pharmaceutical Sciences, Josai International University; 1 Gumyo, Togane, Chiba 283-8555, Japan. \\ Received September 14, 2011; accepted November 8, 2011; published online November 16, 2011
}

The fatty acid profile of hepatic lipid in spontaneously hypertensive rats (SHR)/NDmcr-cp (cp/cp) rats (SHR/NDcp), which offer an animal model of the metabolic syndrome, was characterized by comparing those in Wistar Kyoto rats (WKY), SHR, stroke-prone spontaneously hypertensive rats (SHRSP) and SHR/ NDmcr-cp $(+/+)$ rats $(S H R / N D+)$. Hierarchical clustering analysis revealed that SHR/NDcp and the other four strains and/or substrains of rats were clearly disparate in fatty acid profile of hepatic lipid and that the disparity observed was due to the drastic increases in the mass of monounsaturated fatty acids, especially palmitoleic acid and oleic acid, in the liver of SHR/NDcp. Activities of stearoyl-CoA desaturase (SCD) and palmitoyl-CoA chain elongase in hepatic microsomes of SHR/NDcp were markedly higher than those of WKY, SHR, SHRSP and SHR/ND+. Activities of palmitoleoyl-CoA chain elongase in the liver of SHR/ NDcp were also higher, but to a lesser extent. mRNA levels of SCD1 and elongation of very long-chain fatty acids (Elovl6), but not Elovl5, in the liver of SHR/NDcp were remarkably higher than those of the other four groups of rats. These results suggest that the enhanced expressions of SCD1 and Elovl6 induced abnormalities in fatty acid profile in the liver of SHR/NDcp.

Key words fatty acid profile; palmitoleic acid; oleic acid; liver; SHR/NDmcr-cp ( $c p / c p)$ rat

Obesity, hypertension, hyperlipidemia and hyperglycemia are risk factors for cardiovascular diseases. Patients who have two or more of these risk factors are susceptible to cardiovascular diseases; these states are called the metabolic syndrome. To explore protection against and treatment of the metabolic syndrome, studies on the pathophysiology and metabolic consequences of the metabolic syndrome are required using pertinent animal models. Spontaneously hypertensive rats (SHR)/NDmcr-cp (cp/cp) rats (SHR/NDcp) spontaneously develop obesity and hypertension, and display dyslipidemia and hyperglycemia, the values of body weight, systolic blood pressure, serum triglyceride and blood glucose in SHR/NDcp being 1.43, 1.65, 25.4 and 1.25 times, respectively, compared with those in Wistar Kyoto rats (WKY) (control rats) at 19 or 20 weeks of age. ${ }^{1)}$ Thus, they provide a useful model of the metabolic syndrome. ${ }^{2,3)}$ Hypertensive rats that spontaneously developed as a colony from normotensive WKY, were named SHR. ${ }^{4}$ By crossing SHR with Sprague-Dawley (SD) rats, obese spontaneously hypertensive rats, Koletsky strain, were obtained; this strain of rats is heterozygous for the $c p$ gene. $^{5)}$ To eliminate the noncorpulent genes of the Koletsky strain, the rats were mated with SHR, and a congenic rat strain (SHR/National Institutes of Health (NIH)-corpulent) was developed. ${ }^{6}$ SHR/NDcp are a substrain of SHR/NIHcorpulent rats. With regard to metabolic abnormalities as to hypertension, hyperinsulinemia and hyperglycemia in SHR/ NDcp, many studies have been conducted. On the other hand, information on dyslipidemia in SHR/NDcp is very limited. ${ }^{1,7}$ To our knowledge, no information is available on fatty acid metabolism in the liver, in particular. To establish SHR/NDcp as a useful model of the metabolic syndrome, it is necessary to document characteristics of fatty acid metabolism in the

${ }^{\dagger}$ Present address: Omiya-Kyoritsu Hospital; 1550 Katayanagi, Minumaku, Saitama 337-0024, Japan. liver, because hepatic fatty acid composition is considered to be a determinant of insulin sensitivity that acts independently of cellular energy balance and stress. ${ }^{8)}$ The aims of this study are (i) to characterize the fatty acid profile in the liver of SHR/NDcp and (ii) to demonstrate the mechanism underlying the abnormalities in hepatic fatty acid composition.

\section{MATERIALS AND METHODS}

Chemicals The following materials were obtained from the indicated commercial sources: $\left[2-{ }^{14} \mathrm{C}\right]$ malonyl-CoA (56.0 Ci/mol) (GE Healthcare Bio-Sciences Corp., Piscataway, NJ, U.S.A.); palmitoyl-CoA, palmitoleoyl-CoA, stearoyl-CoA, malonyl-CoA and bovine serum albumin (essentially fatty acid-free for enzyme assays and fraction $\mathrm{V}$ for protein assay) (Sigma Aldrich Japan, Tokyo, Japan); nicotinamide adenine dinucleotide reduced (NADH) (Oriental Yeast Co., Tokyo, Japan); nonadecanoic acid (Nu-Chek-Prep Inc., Elysian, MN, U.S.A.). All other chemicals used were of analytical grade.

Animals Fifteen-week-old male WKY, SHR, strokeprone spontaneously hypertensive rats (SHRSP), SHR/NDcp and nonobese hypertensive littermates of SHR/NDcp (SHR/ NDmcr-cp $(+/+)$ rats $(\mathrm{SHR} / \mathrm{ND}+))$ were obtained from SLC (Hamamatsu, Japan). Animals were fed on a standard diet (CE-2, Clea Japan, Tokyo, Japan) ad libitum and allowed free access to water. After acclimatization, rats were killed at the age of 22 weeks under diethyl ether anesthesia, and livers were excised. One part of the liver was frozen in liquid nitrogen and then stored at $-80^{\circ} \mathrm{C}$ for determination of mRNA. The other part of the liver was perfused with ice-cold $0.9 \% \mathrm{NaCl}$. Liver was homogenized with 4 volumes of $0.25 \mathrm{M}$ sucrose $/ 1 \mathrm{~mm}$ ethylenediaminetetraacetic acid (EDTA) $/ 10 \mathrm{~mm}$ Tris- $\mathrm{HCl}(\mathrm{pH} 7.4)$ in a Potter glass-Teflon homogenizer. An aliquot of the homogenates was frozen in liquid nitrogen and stored at $-80^{\circ} \mathrm{C}$ for analyses of fatty acids. The remaining 
Table 1. Sequences of Primers Used for Real-Time PCR

\begin{tabular}{|c|c|c|}
\hline & Primer $\left(5^{\prime}-3^{\prime}\right)$ & Accession No. \\
\hline \multirow[t]{2}{*}{ SCD1 } & F: TCACCTTGAGAGAAGAATTAGCA & J02585 \\
\hline & R: TTCCCATTCCCTTCACTCTGA & \\
\hline \multirow[t]{2}{*}{ SCD2 } & F: TGCACCCCCAGACACTTGTAA & AB032243 \\
\hline & R: GGATGCATGGAAACGCCATA & \\
\hline \multirow[t]{2}{*}{ Fads1 } & F: AACTGGTTTGTGTGGGTGACG & NM_053445 \\
\hline & R: GAGACCCAGTCCACATTCCG & \\
\hline \multirow[t]{2}{*}{ Fads2 } & F: GCCACTTAAAGGGTGCCTCC & ВC081776 \\
\hline & R: TGCAGGCTCTTTATGTCGGG & \\
\hline \multirow[t]{2}{*}{ Elovl1 } & F: AGCACTTCGGATGGTTCGAGT & ВС085795 \\
\hline & R: GAGTGGTGGAAGACATGGAGGA & \\
\hline \multirow[t]{2}{*}{ Elov12 } & F: TATTCTTGCTTGCCCGTGAGA & NM_001109118 \\
\hline & R: CTGCCATTGTTGATCTGCCA & \\
\hline \multirow[t]{2}{*}{ Elov15 } & F: ACCACCATGCCACTATGCTCA & AB071985 \\
\hline & R: GGACGTGGATGAAGCTGTTGA & \\
\hline \multirow[t]{2}{*}{ Elovl6 } & F: AGAACACGTAGCGACTCCGAA & АВ071986 \\
\hline & R: CAAACGCGTAAGCCCAGAAT & \\
\hline \multirow[t]{2}{*}{$\beta$-Actin } & F: TGCAGAAGGAGATTACTGCC & V01217 \\
\hline & R: CGCAGCTCAGTAACAGTCC & \\
\hline
\end{tabular}

homogenates were centrifuged at $18000 \times \boldsymbol{g}$ for $20 \mathrm{~min}$, and the supernatant was centrifuged under the same conditions. The resulting supernatant was centrifuged at $105000 \times \boldsymbol{g}$ for $60 \mathrm{~min}$. The obtained pellet was resuspended in $0.25 \mathrm{M}$ sucrose $/ 0.1 \mathrm{~mm}$ EDTA/10 mm Tris- $\mathrm{HCl}(\mathrm{pH}$ 7.4) and recentrifuged under the same conditions. The resulting pellet was resuspended in a small volume of $0.25 \mathrm{~m}$ sucrose $/ 0.1 \mathrm{~mm}$ EDTA/10 mM Tris- $\mathrm{HCl}$ ( $\mathrm{pH} 7.4$ ) and used as microsomes. All operations were carried out at $0-4^{\circ} \mathrm{C}$. Protein concentrations were determined by the method of Lowry et al. ${ }^{9)}$ using bovine serum albumin as a standard. All animal studies complied with the regulations of the institutional board for animal studies, Josai University.

Quantitative Fatty Acid Profiling After the addition of a known amount of nonadecanoic acid as an internal standard, total lipid was extracted from liver homogenates according to the method of Bligh and Dyer. ${ }^{10)}$ After the solvent of lipid extract was evaporated, $1 \mathrm{~L}$ of $10 \%(\mathrm{w} / \mathrm{v}) \mathrm{KOH} / 90 \%$ methanol was added to the obtained residue, and then the mixture was heated at $80^{\circ} \mathrm{C}$ for $60 \mathrm{~min}$ for saponification under a nitrogen atmosphere. After the addition of $4 \mathrm{~mL}$ of water, non-saponified materials were removed three times by the extraction with $n$-hexane. The aqueous phase was acidified by the addition of $6 \mathrm{M} \mathrm{HCl}$, and free fatty acids were extracted three times with $n$-hexane. The extract was taken to dryness, and to the residue was added $1 \mathrm{~mL}$ of $14 \%(\mathrm{w} / \mathrm{v})$ boron trifluoride/methanol. The mixture was heated at $100^{\circ} \mathrm{C}$ for $10 \mathrm{~min}$ under a nitrogen atmosphere. After the addition of $4 \mathrm{~mL}$ of water, fatty acid methyl esters were extracted three times with $n$-hexane. All solvents used contained $0.005 \%(\mathrm{w} / \mathrm{v})$ butylated hydroxytoluene. The mass and proportion of the fatty acid methyl esters were determined by GLC [Shimadzu GC-2014 (Kyoto, Japan)], equipped with a flame ionization detector and Supelcowax 10 column $(0.53 \mathrm{~mm} \times 30 \mathrm{~m})$.

Enzyme Assays Stearoyl-CoA desaturase (SCD) activity was assayed spectrophotometrically by the method of Oshino et $a{ }^{11)}$ as the stearoyl-CoA stimulated re-oxidation of NADHreduced cytochrome $b_{5}$. The rate of cytochrome $b_{5}$ oxidation was measured by recording the changes in absorbance between 424 and $409 \mathrm{~nm}$ at $30^{\circ} \mathrm{C}$. The cuvette contained $1.2 \mathrm{mg}$ of microsomal protein and $100 \mathrm{~mm}$ Tris- $\mathrm{HCl}(\mathrm{pH} 7.4)$ in a final volume of $3 \mathrm{~mL}$. Cytochrome $b_{5}$ was reduced by adding $2 \mathrm{nmol} \mathrm{NADH}$ and the re-oxidation was recorded. When the re-oxidation was completed, $20 \mathrm{nmol}$ stearoyl-CoA was added, and cytochrome $b_{5}$ was reduced again using $2 \mathrm{nmol}$ NADH. The first-order rate constant for the re-oxidation of NADH-reduced cytochrome $b_{5}$ was calculated as described by Oshino and Sato. ${ }^{12}$ ) The rate constant for the re-oxidation of cytochrome $b_{5}$ was measured in the presence $(k)$ and in the absence $\left(k^{-}\right)$of stearoyl-CoA; the rate constant for SCD was given by $k^{+}=k-k^{-13)}$

Palmitoyl-CoA chain elongase (PCE) and palmitoleoylCoA chain elongase (POCE) were assayed as the activities of microsomal condensation of palmitoyl-CoA or palmitoleoylCoA with malonyl-CoA by measuring the incorporation of $\left[2-{ }^{14} \mathrm{C}\right]$ malonyl-CoA into the exogenous acyl-CoAs essentially according to a method reported previously. ${ }^{14)}$ The assay mixture contained $15 \mathrm{nmol}$ palmitoyl-CoA or palmitoleoyl-CoA, $100 \mathrm{nmol}\left[2-{ }^{14} \mathrm{C}\right]$ malonyl-CoA $(20 \mathrm{nCi}), 12 \mathrm{nmol}$ bovine serum albumin, $0.5 \mu \mathrm{mol} \mathrm{KCN}, 250 \mu \mathrm{g}$ of microsomal protein and $100 \mathrm{~mm}$ Tris- $\mathrm{HCl}(\mathrm{pH} \mathrm{7.4)}$ in a total volume of $0.5 \mathrm{~mL}$. The mixture was incubated under a nitrogen atmosphere at $37^{\circ} \mathrm{C}$ for $4 \mathrm{~min}$, and the reaction was stopped by adding $1 \mathrm{~mL}$ of $10 \% \mathrm{KOH} / 90 \%$ methanol. The mixture was heated at $80^{\circ} \mathrm{C}$ for $30 \mathrm{~min}$ under a nitrogen atmosphere and then acidified by adding $3 \mathrm{~mL}$ of $4 \mathrm{M} \mathrm{HCl}$. The condensation products were extracted four times with $2 \mathrm{~mL}$ of $n$-hexane. The combined $n$-hexane extract was washed with $4 \mathrm{~mL}$ of acidic water, transferred to a counting vial, and dried under nitrogen. The residue was dissolved in toluene-based scintillator, and the radioactivity was measured using a liquid scintillation counter. The control value, which was obtained from the incubation without acyl-CoA, was subtracted to give the net condensation rate for palmitoyl-CoA or palmitoleoyl-CoA.

RNA Extraction and Quantification of Gene Expression Total RNA was isolated from liver tissues using QIAzol reagent and RNeasy kit (QIAGEN, Hilden, Germany). cDNA was synthesized from $500 \mathrm{ng}$ of total RNA with avian myeloblastosis virus reverse transcriptase (Takara, Shiga, Japan). Polymerase chain reaction (PCR) amplification was carried out using SYBR Premix EX Taq (2x) (Perfect Real Time, Takara, Shiga, Japan). The amplification and detection were performed with Applied Biosystems 7500 real time PCR system (Life Technologies Corp., Carlsbad, CA, U.S.A.). The thermal cycling program was as follows: $10 \mathrm{~s}$ denaturation step at $95^{\circ} \mathrm{C}$ followed by 50 cycles of $5 \mathrm{~s}$ denaturation at $95^{\circ} \mathrm{C}$, and $34 \mathrm{~s}$ annealing/extension at $60^{\circ} \mathrm{C}$. Melting curve analysis was performed to confirm the real-time PCR products. Changes in gene expression were calculated by using the comparative threshold cycle $\left(C_{\mathrm{t}}\right)$ method. $C_{\mathrm{t}}$ values were first normalized by subtracting the $C_{\mathrm{t}}$ value obtained from $\beta$-actin (control). The sequences of primers used in this study are listed in Table 1 .

Statistical Analyses Homogeneity of variance was established using one-way analysis of variance. When a difference was significant $(p<0.05)$, Scheffé's multiple range test was used as a post-hoc test. The results were considered to be significant if the value of $p$ was $<0.05$. Hierarchical clustering analysis was performed to evaluate where differences existed in the fatty acid profiles by using JMP 5.1 (SAS Institute Japan, Tokyo, Japan). 
Table 2. Fatty Acid Profile of Hepatic Lipid of WKY, SHR, SHRSP, SHR/ND+ and SHR/NDcp

\begin{tabular}{|c|c|c|c|c|c|}
\hline & WKY & SHR & SHRSP & SHR/ND+ & SHR/NDcp \\
\hline & \multicolumn{5}{|c|}{ ( $\mu \mathrm{mol} / \mathrm{g}$ liver $)$} \\
\hline $16: 0$ & $21.73 \pm 2.75^{\mathrm{a}}$ & $23.98 \pm 0.99^{\mathrm{a}}$ & $23.33 \pm 1.63^{\mathrm{a}}$ & $25.01 \pm 1.22^{\mathrm{a}}$ & $72.90 \pm 26.68^{b}$ \\
\hline $16: 1 \mathrm{n}-7$ & $0.84 \pm 0.39^{\mathrm{a}}$ & $1.12 \pm 0.14^{\mathrm{a}}$ & $0.82 \pm 0.26^{\mathrm{a}}$ & $1.16 \pm 0.22^{\mathrm{a}}$ & $21.15 \pm 7.63^{\mathrm{b}}$ \\
\hline $18: 0$ & $14.27 \pm 1.08$ & $13.75 \pm 0.94$ & $15.49 \pm 1.81$ & $14.62 \pm 0.79$ & $13.24 \pm 1.25$ \\
\hline $18: 1 \mathrm{n}-9$ & $6.36 \pm 1.67^{\mathrm{a}}$ & $7.42 \pm 0.60^{\mathrm{a}}$ & $5.17 \pm 0.70^{\mathrm{a}}$ & $8.00 \pm 0.82^{\mathrm{a}}$ & $57.79 \pm 24.76^{b}$ \\
\hline $18: 1 \mathrm{n}-7$ & $3.75 \pm 0.47^{\mathrm{a}}$ & $4.36 \pm 0.38^{\mathrm{a}}$ & $2.36 \pm 0.33^{\mathrm{a}}$ & $3.70 \pm 0.12^{\mathrm{a}}$ & $12.56 \pm 3.78^{\mathrm{b}}$ \\
\hline $18: 2 n-6$ & $17.17 \pm 1.34^{\mathrm{a}}$ & $21.50 \pm 1.51^{\mathrm{a}}$ & $16.45 \pm 1.27^{\mathrm{a}}$ & $20.00 \pm 2.85^{\mathrm{a}}$ & $39.14 \pm 15.21^{\mathrm{b}}$ \\
\hline $18: 3 n-3$ & $0.16 \pm 0.03^{\mathrm{a}}$ & $0.40 \pm 0.07^{\mathrm{a}}$ & $0.25 \pm 0.06^{\mathrm{a}}$ & $0.53 \pm 0.29^{\mathrm{a}}$ & $1.57 \pm 0.58^{\mathrm{b}}$ \\
\hline $20: 3 n-9$ & $0.04 \pm 0.02$ & $0.02 \pm 0.01$ & $0.08 \pm 0.07$ & $0.20 \pm 0.22$ & $0.26 \pm 0.11$ \\
\hline $20: 3 n-6$ & $0.60 \pm 0.03^{\mathrm{a}}$ & $0.74 \pm 0.18^{\mathrm{a}}$ & $0.57 \pm 0.15^{\mathrm{a}}$ & $0.63 \pm 0.08^{\mathrm{a}}$ & $3.30 \pm 1.11^{\mathrm{b}}$ \\
\hline $20: 4 n-6$ & $16.41 \pm 1.75^{\mathrm{ab}}$ & $15.15 \pm 1.11^{\mathrm{ab}}$ & $13.94 \pm 2.44^{\mathrm{ab}}$ & $17.31 \pm 2.60^{\mathrm{a}}$ & $12.79 \pm 1.05^{\mathrm{b}}$ \\
\hline $20: 5 n-3$ & $0.76 \pm 0.35^{\mathrm{ab}}$ & $0.89 \pm 0.22^{\mathrm{ab}}$ & $0.93 \pm 0.46^{\mathrm{ab}}$ & $0.61 \pm 0.08^{\mathrm{a}}$ & $1.59 \pm 0.58^{\mathrm{b}}$ \\
\hline $22: 5 n-3$ & $1.23 \pm 0.34$ & $2.00 \pm 1.13$ & $1.25 \pm 0.30$ & $1.39 \pm 0.05$ & $2.35 \pm 0.94$ \\
\hline $22: 6 n-3$ & $4.28 \pm 0.52$ & $4.32 \pm 0.48$ & $5.08 \pm 0.48$ & $4.42 \pm 0.36$ & $5.22 \pm 1.05$ \\
\hline Total & $87.58 \pm 6.87^{\mathrm{a}}$ & $95.65 \pm 3.88^{\mathrm{a}}$ & $85.72 \pm 3.20^{\mathrm{a}}$ & $97.57 \pm 7.61^{\mathrm{a}}$ & $243.84 \pm 83.25^{\mathrm{b}}$ \\
\hline Total ( $\mu \mathrm{mol} /$ liver $)$ & $1247.13 \pm 268.36^{\mathrm{a}}$ & $1439.66 \pm 121.08^{\mathrm{a}}$ & $1089.95 \pm 80.93^{\mathrm{a}}$ & $1517.02 \pm 113.12^{\mathrm{a}}$ & $7707.14 \pm 3742.40^{b}$ \\
\hline
\end{tabular}

Values represent mean \pm S.D. for 4 or 5 rats. Differences in horizontal means without a common superscript (a,b) are significant $(p<0.05)$. If no superscript appears, the differences in the means are not siginificant $(p>0.05)$. The fatty acids are designated by the numbers of carbon atoms and double bonds; palmitic acid, $16: 0$; palmitoleic acid, 16:1n-7; stearic acid, 18:0; oleic acid, 18:1 n-9; cis-vaccenic acid, 18:1n-7; linoleic acid, 18:2n-6; $\alpha$-linolenic acid, 18:3n-3; 8,11,14-eicosatrienoic acid, $20: 3 \mathrm{n}-6 ; 5,8,11$-eicosatrienoic acid, 20:3n-9; arachidonic acid, 20:4n-6; 5,8,11,14,17-eicosapentaenoic acid, 20:5n-3; 7,10,13,16,19-docosapentaenoic acid, 22:5n-3; 4,7,10,13,16,19-docosahexaenoic acid, $22: 6 n-3$.
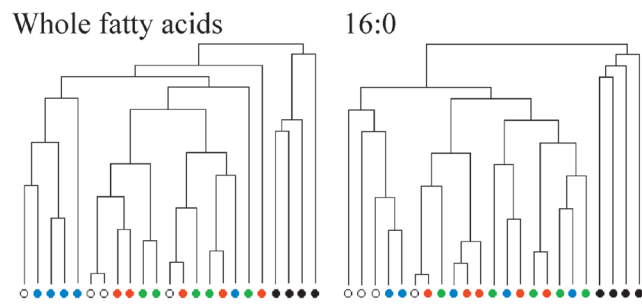

$16: 1 n-7$

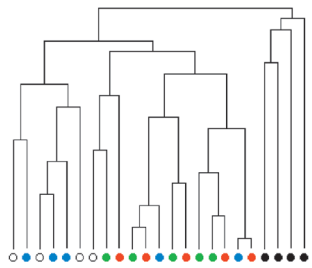

$18: 0$

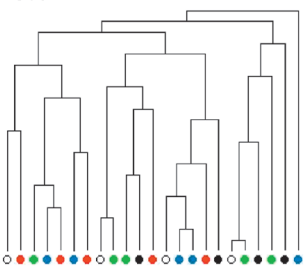

$18: \ln -9$

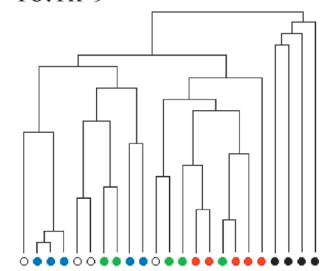

$20: 3 n-9$
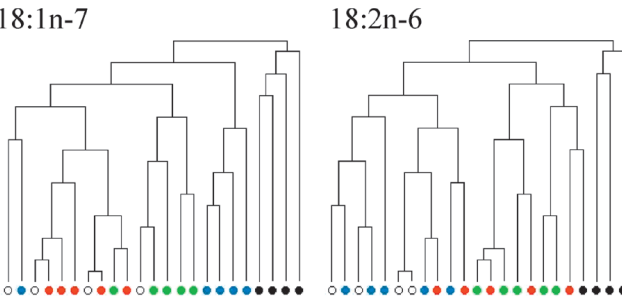

$18: 3 n-3$
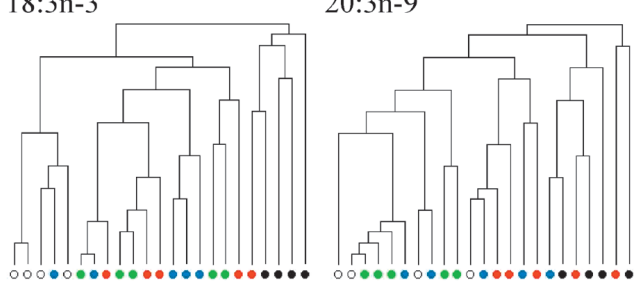

$20 \cdot 3 n-6$

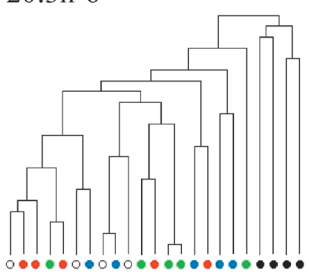

$20: 4 n-6$

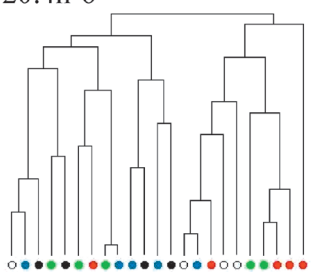

20:5n-3

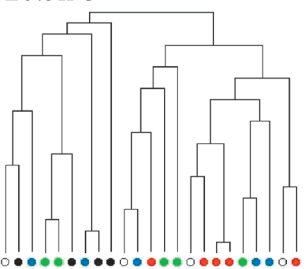

$22: 5 n-3$

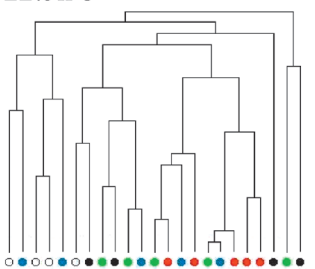

$22: 6 n-3$

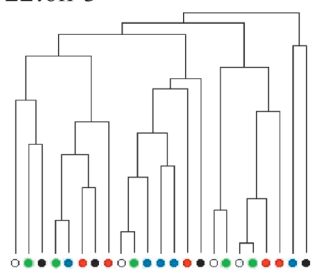

Fig. 1. Hepatic Fatty Acid Profiling

Dendrograms of hierarchical clustering of fatty acids were calculated on the bases of fatty acid profiles of Table 2. O, WKY; $\bullet$, SHR; $\bullet$, SHRSP; $\bullet$ SHR/ND+; SHR/NDcp.

\section{RESULTS}

Characteristic Profile of Fatty Acid in the Liver of SHR/NDcp Table 2 shows the fatty acid profile of hepatic lipid of WKY, SHR, SHRSP, SHR/ND+ and SHR/NDcp. On the basis of the data of Table 2, hierarchical clustering was used to evaluate where the largest differences existed in the fatty acid profiles (Fig. 1). The results show that the lower on the tree the branch is, the more similar the group; alternatively, the higher the branch is, the more disparate the groups. Hierarchical clustering for all kinds of fatty acid species as variables showed that SHR/NDcp and the other four groups 


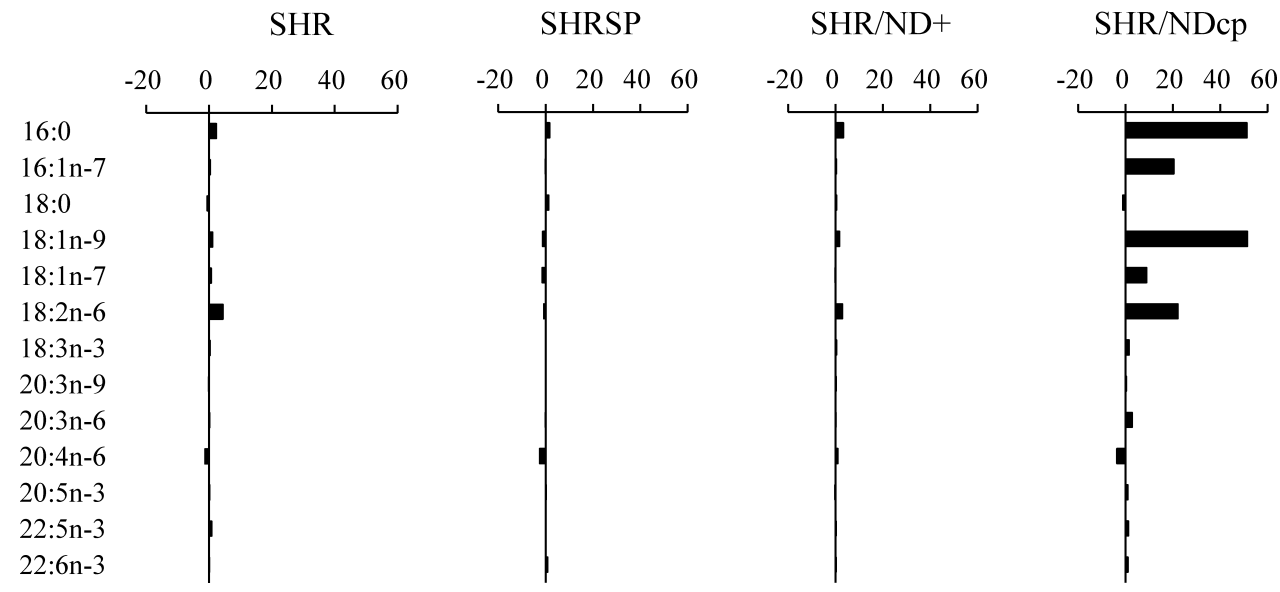

Fig. 2. Difference of Fatty Acids in Hepatic Lipid between WKY and the Other Four Groups, SHR, SHRSP, SHR/ND+ and SHR/NDcp

With regard to each fatty acid, differences in hepatic content ( $\mu \mathrm{mol} / \mathrm{g}$ liver) between the means of WKY and those of the other four groups of rats were calculated from the data of Table 2 .

(WKY, SHR, SHRSP and SHR/ND+) were clearly disparate in profile among all kinds of fatty acids in the liver. Moreover, there was no disparity in profile of all kinds of fatty acids in the liver among WKY, SHR, SHRSP and SHR/ND+. Among the individual fatty acids examined, $16: 0,16: 1 \mathrm{n}-7,18: 1 \mathrm{n}-9$, $18: 1 n-7$ and $20: 3 n-6$ were responsible for the disparity in profile of all kinds of fatty acids between SHR/NDcp and the other four groups of rats. Striking enrichments of particular fatty acids, 16:1n-7 and 18:1n-9, were observed in the liver of SHR/NDcp (Fig. 2). Body weights and liver weights of SHR/NDcp were 1.4 and 2.1 times, respectively, greater than those of WKY, but no notable difference was observed in body weights and liver weights among WKY, SHR, SHRSP and SHR/ND+ (unpublished data), so that total masses (mol/ whole liver) of these monounsaturated fatty acids (MUFAs) were additionally higher. There were no fatty acids of which contents (mol/g liver) in the liver of SHR/NDcp were lower than those of WKY, SHR, SHRSP and SHR/ND+.

Activities of Enzymes Involved in Formation of MUFA Species in the Liver Microsomal activities of SCD, PCE and POCE in the livers were determined (Fig. 3). SCD activities in the liver of SHR/NDcp were 4.6 times higher than those of WKY, and there was no significant difference in SCD activities among WKY, SHR, SHRSP and SHR/ND+ (Fig. 3A). The activity of PCE in microsomes of the liver of SHR/ NDcp was 5.7 times greater than that of WKY, and no significant differences were observed in the activities of PCE among the other four groups of rats (Fig. 3B). As for POCE, the activity in hepatic microsomes of SHR/NDcp was 1.8 times higher than that of WKY, no difference being observed in the activities among WKY, SHR and SHR/ND+ (Fig. 3C). The activity of POCE in the liver of SHRSP was 0.44 times lower than that of WKY.

Expression of Genes Encoding Fatty Acid Desaturases and Elongases in the Liver The levels of mRNA encoding enzymes that are involved in desaturation and chain elongation of fatty acids in the liver of rats, were determined by realtime PCR (Figs. 4, 5). With regard to the expression of genes encoding desaturases, the levels of SCD1 mRNA of which translation product catalyzes conversion of $16: 0$ and 18:0 to $16: 1 \mathrm{n}-7$ and 18:1n-9, respectively, in the liver of SHR/NDcp were markedly higher than those of WKY, SHR, SHRSP and
SHR/ND+, and the extent of the enhancement of expression was 5.5 times the level in WKY (Fig. 4A). There was no notable difference in the expression of SCD1 gene among WKY, SHR, SHRSP and SHR/ND+ (Fig. 4A). In contrast, the mRNA levels of SCD2 in the liver were slightly, but significantly, lower in SHR/NDcp than in WKY and SHR/ND+ (Fig. 4B). The levels of fatty acid desaturase (Fads) 1 mRNA encoding $\Delta 5$ desaturase, which converts $20: 3 n-6$ to $20: 4 n-6$, in SHR/NDcp were lower than those in the other four groups of rats, the value being 0.46 times lower than that of WKY (Fig. 4C). There was no significant difference in the expression of Fads 2 mRNA of which product is $\Delta 6$ desaturase that converts $18: 2$ n- 6 to $18: 3$ n- 6 between WKY and SHR/NDcp, whereas the levels of Fads2 mRNA in SHR was 0.39 times lower than that in WKY (Fig. 4D). Of the seven elongases identified in rats, four elongases are demonstrated to be expressed in the liver of rats; elongation of very long-chain fatty acids (Elovl)5 mRNA appears to be expressed in a high abundance, whereas the relative abundances of mRNAs encoding Elovl1, Elovl2 and Elovl6 are far lower than that of Elov15. ${ }^{15}$ The differences are reported in substrate preference of these elongase subtypes. ${ }^{16)}$ Namely, translation product of Elovl1 elongates a broad array of saturated and monounsaturated fatty acids; Elovl2 protein has a narrow substrate preference for polyunsaturated fatty acids with carbon chain length of 2022; Elovl5 product converts $16: 1 \mathrm{n}-7$, but not $16: 0$, to $18: 1 \mathrm{n}-7$ as well as $18: 3 n-6$ to $20: 3 n-6$; Elovl6 protein has a narrow substrate preference for saturated and monounsaturated fatty acids with carbon chain length of $12-16$. With regard to the expression of genes encoding elongases, the level of mRNA encoding Elovll in SHR/NDcp was significantly lower than those in WKY and SHR/ND+ (Fig. 5A). The mRNA levels of Elovl2 in SHR, SHR/ND+ and SHR/NDcp were significantly lower than that in WKY (Fig. 5B). The mRNA level of Elovl5 in the liver of SHRSP was slightly, but significantly, higher than that of WKY; there was no significant difference in the expression of the gene encoding Elovl5 among the other four groups of rats (Fig. 5C). The mRNA level of Elovl6 in the liver of SHR/NDcp was 81 times higher than that in WKY, and, on the other hand, no significant difference was observed in the levels among the other four groups of rats (Fig. 5D). 

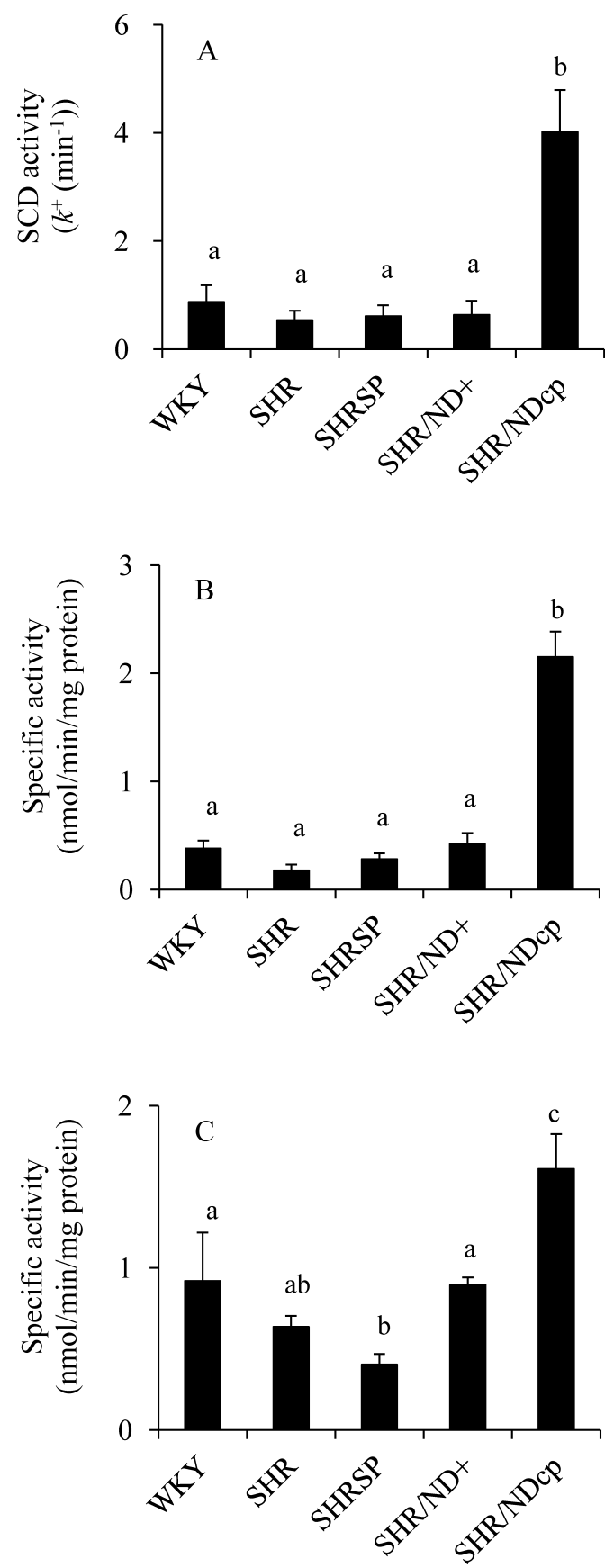

Fig. 3. Activities of SCD, PCE and POCE in Hepatic Microsomes

A, SCD; B, PCE; C, POCE. Values represent mean \pm S.D. for 4 or 7 rats. ${ }^{\mathrm{a}-\mathrm{c}}$ Differences without a common superscript are statistically significant $(p<0.05)$.

\section{DISCUSSION}

To understand how dissimilar SHR/NDcp are in the fatty acid profile in hepatic lipid compared with WKY, SHR, SHRSP and SHR/ND+, hierarchical clustering was employed in the present study. Hierarchical clustering is a widely used method for cluster analysis which consists in grouping data objects into a tree of clusters based on their distance (or similarity). Visualizing this tree provides a useful summary of the data. The clustering analyses clearly showed that SHR/NDcp and the other four strains and/or substrains of rats, WKY, SHR, SHRSP and SHR/ND+, were apparently disparate in fatty acid profile in hepatic lipid and that the disparity was accounted for by the selective elevations of contents of $16: 0$, $16: 1 \mathrm{n}-7,18: 1 \mathrm{n}-9,18: 1 \mathrm{n}-7$ and $20: 3 \mathrm{n}-6$ in the liver of SHR/ NDcp. Of these fatty acid species, the extents of the elevation of the individual fatty acids in SHR/NDcp versus WKY were predominant in $16: 1 \mathrm{n}-7(20.3 \mu \mathrm{mol} / \mathrm{g}$ liver; 25.2-fold), followed by $18: 1 \mathrm{n}-9(51.4 \mu \mathrm{mol} / \mathrm{g}$ liver; 9.1 -fold $)$ and $20: 3 \mathrm{n}-6$ ( $2.7 \mu \mathrm{mol} / \mathrm{g}$ liver; 5.5 -fold). Although $18: 1 \mathrm{n}-7$ is one of the MUFAs that are capable of being synthesized in the liver, its elevation $(8.8 \mu \mathrm{mol} / \mathrm{g}$ liver; 3.3 -fold $)$ in SHR/NDcp was far lower than that of 18:1n-9. Thus, SHR/NDcp displayed abnormalities in fatty acid composition of the liver. Pathways of biosynthesis of the MUFAs in the liver are shown in Fig. 6. 18:1n-9 is synthesized from 16:0 via 18:0. Namely, 16:0 is elongated by $\mathrm{PCE}^{17,18)}$ and subsequently desaturated by $\Delta 9$ desaturation to yield $18: 1 n-9 .{ }^{19)}$ The present study showed that the activities of PCE and SCD in hepatic microsomes of SHR/ NDcp were much higher than those of WKY, SHR, SHRSP and SHR/ND+; PCE and SCD activities in SHR/NDcp were 5.7 and 4.6 times, respectively, higher than those in WKY. These results enable us to elucidate the preferential elevation of $18: 1 \mathrm{n}-9$ in the liver of SHR/NDcp. It is considered that the enzyme that catalyzes $\Delta 9$ desaturation of $16: 0$ is also $\left.\mathrm{SCD},{ }^{19}\right)$ and that $18: 1 \mathrm{n}-7$ is synthesized from $16: 0$ by sequential $\Delta 9$ desaturation and palmitoleoyl-CoA chain elongation. ${ }^{18,20)}$ The present study showed that the POCE activity in hepatic microsomes of SHR/NDcp was 1.8 times higher than that of WKY. It is likely, therefore, that the enrichments of these MUFAs in the liver were mostly due to the increases in the activities of microsomal SCD, PCE and POCE in the liver of SHR/NDcp. These results well account for the facts that the elevation of hepatic content of $16: 1 \mathrm{n}-7$ was striking and that of $18: 1 \mathrm{n}-7$ was moderate in the liver of SHR/NDcp.

Two genes encoding SCD in the liver of rats have been cloned and characterized so far. ${ }^{21)}$ Of the SCD subtypes, SCD1 is expressed at a high level in the liver, ${ }^{21)}$ and its expression is regulated by a number of nutritional and hormonal factors. ${ }^{22}$ SCD1 is considered to be important in lipid homeostasis and body weight regulation. ${ }^{22-25)}$ On the other hand, in the liver of rats, SCD2 is also expressed, but less than SCD1. ${ }^{21)}$ The present study demonstrated that gene expression of SCD1 in the liver of SHR/NDcp was 5.5 times higher than that of WKY, whereas there was no notable difference in the expression of the gene encoding SCD2 among the five groups of rats. With regard to fatty acid elongase, 7 isoforms (Elovll ca. 7) have been identified so far. ${ }^{26,27)}$ Of the 7 isoforms, rat liver expresses four isoforms: Elovl1, Elovl2, Elov15 and Elovl6. ${ }^{15)}$ The expression of only Elovl6 was strikingly high in the liver of SHR/NDcp; on the other hand, no differences were observed in the expression of Elovl5 mRNA between SHR/NDcp and the other four groups of rats. The present results are in accordance with the previous findings that 16:0 is elongated by Elovl6 and desaturated by SCD1 to yield $18: 1 \mathrm{n}-9 .{ }^{16,28)}$ Moreover, Elovl6 plays a crucial role in the elongation of $16: 1 \mathrm{n}-7$ to $18: 1 \mathrm{n}-7$, as well as in that of $16: 0$ to $18: 0 .^{8,16,28)}$ Elov15 is demonstrated to participate in POCE, but little to convert $16: 0$ to $18: 0{ }^{16,28)}$ Interestingly, however, our present study showed that the increase in the activity of POCE in SHR/NDcp was 1.8 times compared to that of WKY whereas PCE activity in SHR/NDcp was 5.7 times, and the expression of Elovl5 was not enhanced in the liver of SHR/NDcp, despite 

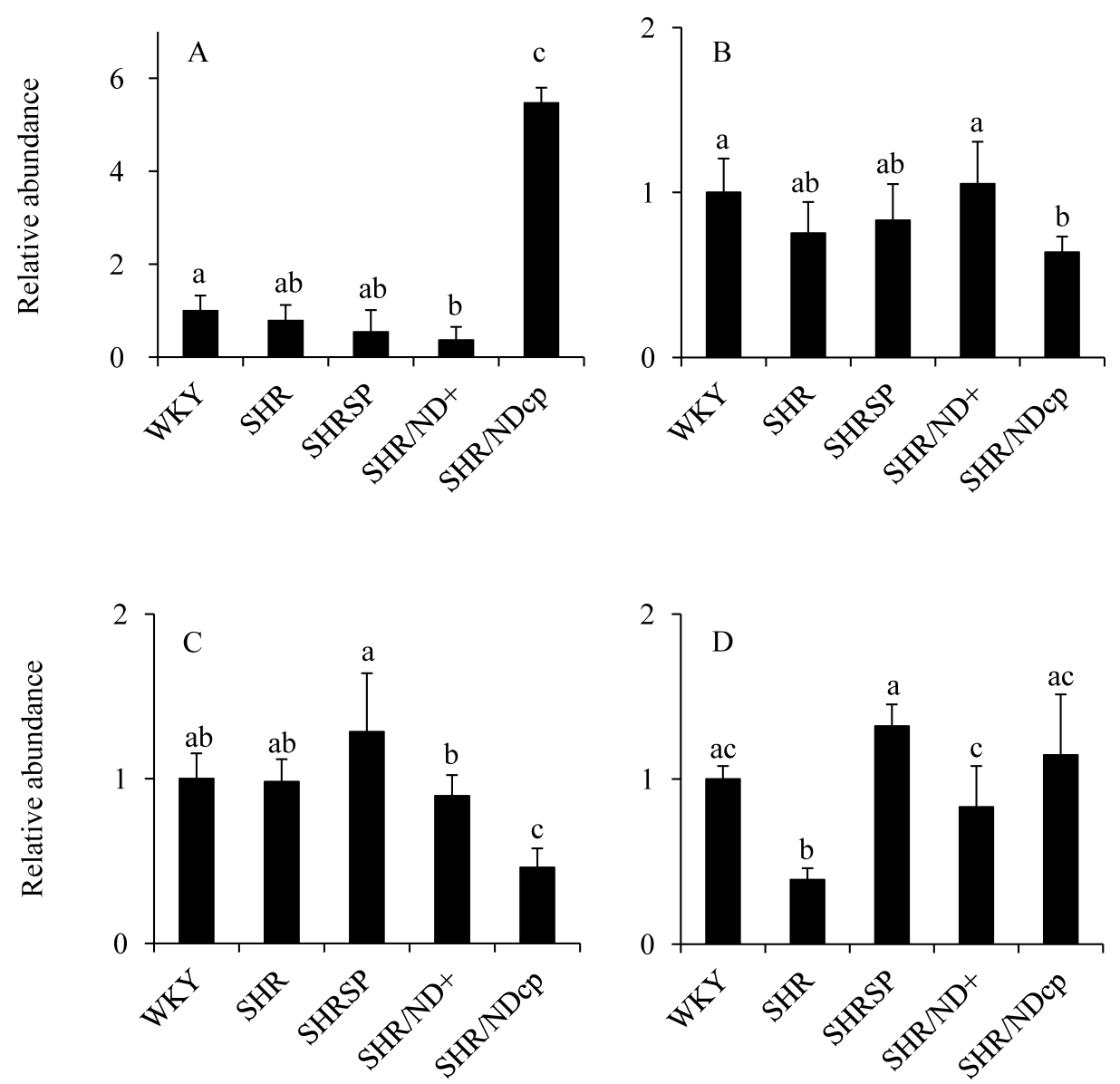

Fig. 4. Levels of mRNA Encoding Desaturases in the Liver

A, SCD1; B, SCD2; C, Fads1; D, Fads2. Values represent mean \pm S.D. for 4 or 9 rats. ${ }^{a-c}$ Differences without a common superscript are statistically significant $(p<0.05)$.

the fact that expression of Elovl6 was strikingly augmented. These results obtained from SHR/NDcp are distinct from the findings that obesity enhances expression of Elovl5 in the liver of obese mice (C57BL/6J-Lepob/ob) as well as Elovl6. $\left.{ }^{16}\right)$ These findings, taken together, suggest that POCE in the liver of SHR/NDcp is composed of at least two elongases with a relatively large proportion of Elovl5 product and a relatively small proportion of Elovl6 product and that the elevated activity of POCE is due to the increase in Elovl6 product in the liver of SHR/NDcp. These present results demonstrated that coordinate alterations in the expression of genes encoding SCD, Elovl6 and Elovl5 produce the disordered balance in n-9 and n-7 MUFA species in the liver of SHR/NDcp.

$20: 3 n-6$ is one of the intermediates of $20: 4 n-6$ formation; $20: 4 n-6$ is considered to be synthesized from $18: 2 \mathrm{n}-6$ via the pathway of $18: 2 \mathrm{n}-6 \rightarrow 6,9,12$-octadecatrienoic acid (18:3n-6) $\rightarrow 20: 3 \mathrm{n}-6 \rightarrow 20: 4 \mathrm{n}-6 .{ }^{29)}$ The process of $18: 2 \mathrm{n}-6 \rightarrow 18: 3 \mathrm{n}-6$ is catalyzed by $\Delta 6$ desaturase, which is encoded by Fads2. ${ }^{29,30)}$ The elongase responsible for the conversion of $18: 3 n-6$ to 20:3n-6 is encoded by Elov15. ${ }^{16)} \Delta 5$ Desaturase that catalyzes the final step of $20: 4 n-6$ formation is encoded by Fads1. ${ }^{29)}$ The process of $18: 2 n-6$ to $18: 3 n-6$ is considered to be the rate-limiting step of $20: 4 n-6$ synthesis in rat livers with physiologically normal states. ${ }^{29)}$ However, the difference between the activities of $\Delta 6$ desaturation $(18: 2 n-6 \rightarrow 18: 3 n-6)$ and $\Delta 5$ desaturation $(20: 3 n-6 \rightarrow 20: 4 n-6)$ is not significantly large, and the process of $18: 3 n-6 \rightarrow 20: 3 n-6$ is much faster than that of either $\Delta 5$ desaturation or $\Delta 6$ desaturation in rat livers. ${ }^{29)}$ The present study showed that the expression of Fads1 was 0.46 times lower in SHR/NDcp than in WKY, whereas there was no difference in the expressions of Fads2 and Elovl5 between SHR/NDcp and WKY. It seems likely, therefore, that the rate-limiting step of $20: 4 \mathrm{n}-6$ formation was changed from the process of $\Delta 6$ desaturation to that of $\Delta 5$ desaturation, so that 20:3n-6 was accumulated in hepatic lipid of SHR/NDcp.

The physiological or pathological significance of the elevations of hepatic contents of 16:1n-7 and 18:1n-9 in SHR/ NDcp has not been clarified yet. Two possibilities, however, are conceivable for the accumulation of 18:1n-9. One possibility is that accumulation of $18: 1 \mathrm{n}-9$ as intracellular lipid caused dyslipidemia such as hepatosteatosis, hypertriglyceridemia and obesity in SHR/NDcp. Intracellular accumulation of lipid in organs is considered to be one of the most likely causes of dysfunction of the organ with regards to insulin resistance. $^{31)} 18: 1 \mathrm{n}-9$, which is synthesized from 18:0 by SCD, is a major component of triglyceride, and deficiency of the SCD1 gene in mice protects against hypertriglyceridemia, ${ }^{32)}$ obesity, ${ }^{33,34)}$ and postprandial plasma insulin. ${ }^{34,35)}$ Moreover, mice deficient in Elovl6 showed marked protection from hyperinsulinemia, hyperglycemia and hyperleptinemia. ${ }^{8}$ An alternative possibility is that the formation of $18: 1 \mathrm{n}-9$ was increased in the liver of SHR/NDcp to prevent the function of the tissue from endoplasmic reticulum (ER) stress by saturated fatty acids such as 18:0 and 16:0, which are known 

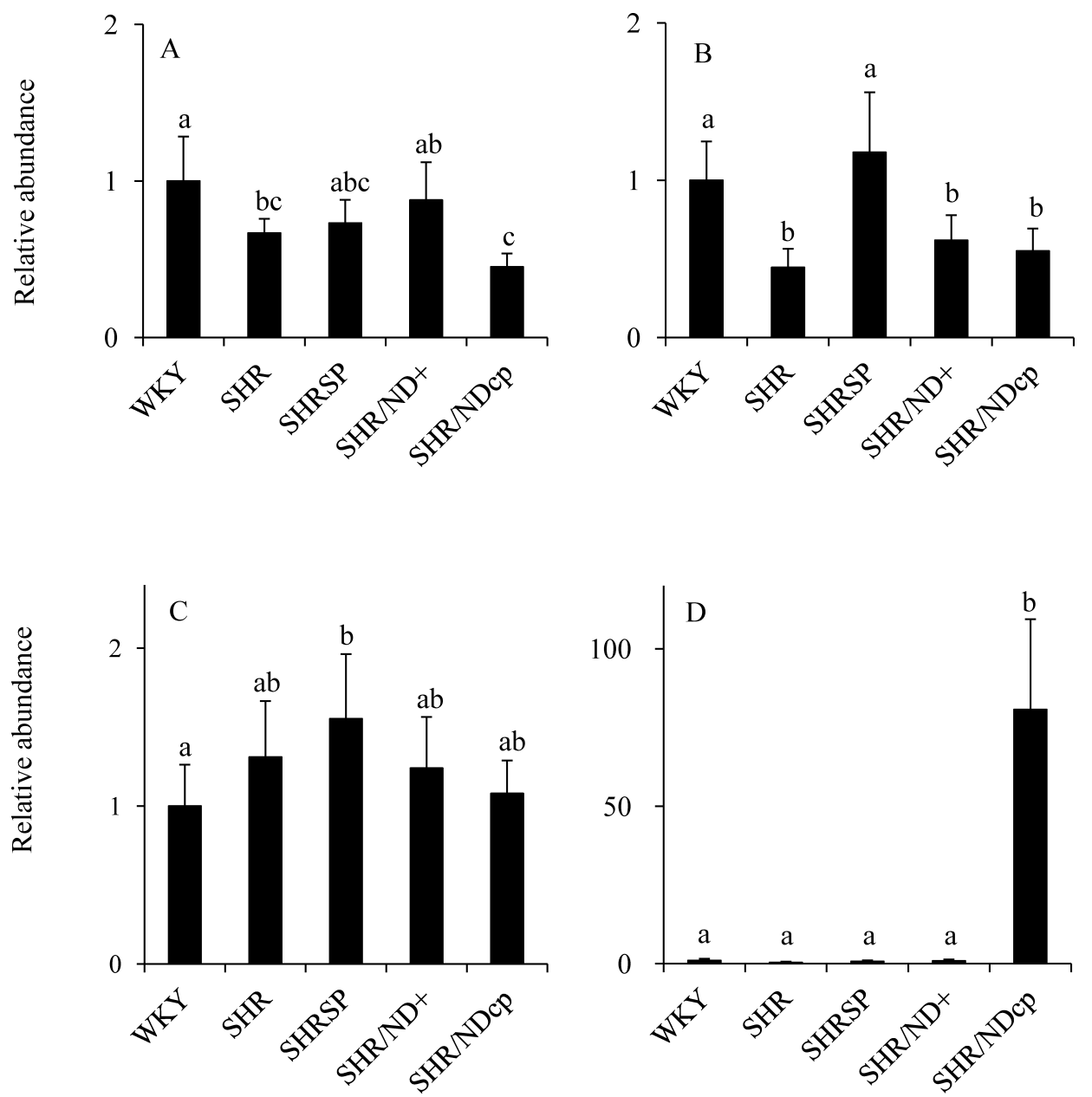

Fig. 5. Levels of mRNA Encoding Elongases in the Liver

A, Elov11; B, Elov12; C, Elov15; D, Elovl6. Values represent mean \pm S.D. for 4 or 9 rats. ${ }^{a-c}$ Differences without a common superscript are statistically significant $(p<0.05)$.

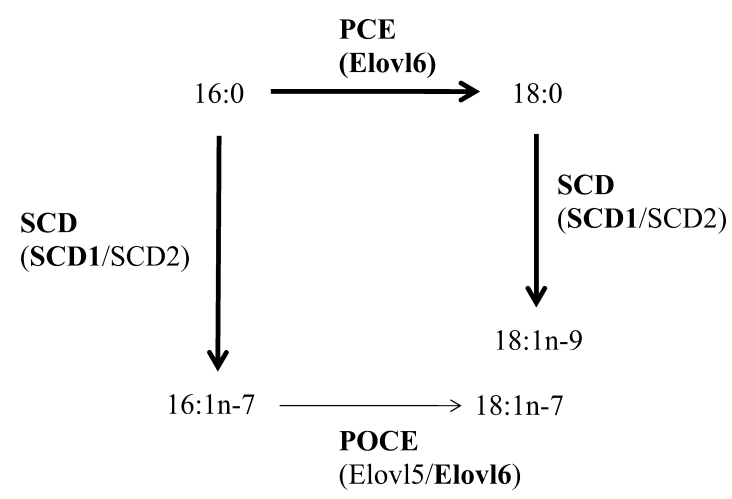

Fig. 6. Diagram of Enzymes Regulating Synthesis of $16: 1 \mathrm{n}-7,18: 1 \mathrm{n}-7$ and $18: 1 \mathrm{n}-9$

to cause ER stress in the liver. ${ }^{36,37)} 18: 1 \mathrm{n}-9$ is preferentially incorporated into triglyceride, and triglyceride is probably the least toxic form in which the surplus of lipid, in particular 18:0 and 16:0, can be sequestered and may, at least in the short term, actually protect against severe metabolic trauma. ${ }^{38)}$ With regard to $16: 1 \mathrm{n}-7$, recent studies have suggested that this specific fatty acid species has significant impact on the insulin sensitivity of the liver and whole body. ${ }^{839)}$ In this regard, therefore, it is considered that hepatic fatty acid com- position is likely to be a determinant of insulin sensitivity that acts independently of cellular energy balance and stress. ${ }^{8)}$

In conclusion, SHR/NDcp provides an excellent example of defective liporegulation and is an interesting model to study the physiological or pathological significance of disturbances in fatty acid metabolism in metabolic syndrome. Currently, however, the detailed mechanism underlying this disorder in regulation of fatty acid synthesis remains to be resolved.

Acknowledgments This work was supported by a Grantin-Aid for Scientific Research from the Ministry of Education, Culture, Sports, Science and Technology of Japan.

\section{REFERENCES}

1) Kawai K, Sakairi T, Harada S, Shinozuka J, Ide M, Sato H, Tanaka M, Toriumi W, Kume E. Diet modification and its influence on metabolic and related pathological alterations in the SHR/NDmcrcp rat, an animal model of the metabolic syndrome. Exp. Toxicol. Pathol., in press (2010).

2) Kagota S, Yamaguchi Y, Tanaka N, Kubota Y, Kobayashi K, Nejime N, Nakamura K, Kunitomo M, Shinozuka K. Disturbances in nitric oxide/cyclic guanosine monophosphate system in SHR/ NDmcr-cp rats, a model of metabolic syndrome. Life Sci., 78, 1187-1196 (2006).

3) Hussein G, Nakagawa $T$, Goto $H$, Shimada $Y$, Matsumoto K, 
Sankawa U, Watanabe H. Astaxanthin ameliorates features of metabolic syndrome in SHR/NDmcr-cp. Life Sci., 80, 522-529 (2007).

4) Okamoto K, Aoki K. Development of a strain of spontaneously hypertensive rats. Jpn. Circ. J., 27, 282-293 (1963).

5) Koletsky S. Obese spontaneously hypertensive rats - a model for study of atherosclerosis. Exp. Mol. Pathol., 19, 53-60 (1973).

6) Michaelis OE 4th, Ellwood KC, Judge JM, Schoene NW, Hansen CT. Effect of dietary sucrose on the SHR/N-corpulent rat: a new model for insulin-independent diabetes. Am. J. Clin. Nutr., 39, 612-618 (1984).

7) Michihara A, Anraku M, Abe A, Kinoshita H, Kamizaki Y, Tomida $\mathrm{H}$, Akasaki K. Comparison of receptors and enzymes regulating cholesterol levels in liver between SHR/NDmcr-cp rats and normotensive Wistar Kyoto rats at ten weeks of age. Biol. Pharm. Bull., 34, 1116-1119 (2011).

8) Matsuzaka T, Shimano H, Yahagi N, Kato T, Atsumi A, Yamamoto $\mathrm{T}$, Inoue N, Ishikawa M, Okada S, Ishigaki N, Iwasaki H, Iwasaki Y, Karasawa T, Kumadaki S, Matsui T, Sekiya M, Ohashi K, Hasty AH, Nakagawa Y, Takahashi A, Suzuki H, Yatoh S, Sone H, Toyoshima H, Osuga J, Yamada N. Crucial role of a long-chain fatty acid elongase, Elovl6, in obesity-induced insulin resistance. Nat. Med., 13, 1193-1202 (2007).

9) Lowry OH, Rosebrough NJ, Farr AL, Randall RJ. Protein measurement with the Folin phenol reagent. J. Biol. Chem., 193, 265-275 (1951).

10) Bligh EG, Dyer WJ. A rapid method of total lipid extraction and purification. Can. J. Biochem. Physiol., 37, 911-917 (1959).

11) Oshino $\mathrm{N}$, Imai $\mathrm{Y}$, Sato R. A function of cytochrome $b_{5}$ in fatty acid desaturation by rat liver microsomes. J. Biochem., 69, 155-167 (1971).

12) Oshino N, Sato R. Stimulation by phenols of the reoxidation microsomal bound cytochrome $b_{5}$ and its implication to fatty acid desaturation. J. Biochem., 69, 169-180 (1971).

13) Kawashima Y, Kozuka H. Increased activity of stearoyl-CoA desaturation in liver from rat fed clofibric acid. Biochim. Biophys. Acta, 713, 622-628 (1982).

14) Nagi MN, Cook L, Suneja SK, Osei P, Cinti DL. Spectrophotometric assay for the condensing enzyme activity of the microsomal fatty acid chain elongation system. Anal. Biochem., 179, 251-261 (1989).

15) Wang Y, Botolin D, Christian B, Busik J, Xu J, Jump DB. Tissuespecific, nutritional, and developmental regulation of rat fatty acid elongases. J. Lipid Res., 46, 706-715 (2005).

16) Wang Y, Botolin D, Xu J, Christian B, Mitchell E, Jayaprakasam B, Nair MG, Peters JM, Busik JV, Olson LK, Jump DB. Regulation of hepatic fatty acid elongase and desaturase expression in diabetes and obesity. J. Lipid Res., 47, 2028-2041 (2006).

17) Nakagawa M, Kawashima $Y$, Uchiyama M. Some factors affecting chain elongation of palmityl-CoA in rat liver microsomes. Chem. Pharm. Bull., 24, 46-51 (1976).

18) Prasad MR, Nagi MN, Ghesquier D, Cook L, Cinti DL. Evidence for multiple condensing enzymes in rat hepatic microsomes catalyzing the condensation of saturated, monounsaturated, and polyunsaturated acyl coenzyme A. J. Biol. Chem., 261, 8213-8217 (1986).

19) Enoch HG, Catalá A, Strittmatter P. Mechanism of rat liver microsomal stearyl-CoA desaturase. Studies of the substrate specificity, enzyme-substrate interactions, and the function of lipid. J. Biol. Chem., 251, 5095-5103 (1976).

20) Cinti DL, Cook L, Nagi MN, Suneja SK. The fatty acid chain elongation system of mammalian endoplasmic reticulum. Prog. Lipid Res., 31, 1-51 (1992).

21) Mihara K. Structure and regulation of rat liver microsomal stearoylCoA desaturase gene. J. Biochem., 108, 1022-1029 (1990).

22) Ntambi JM, Miyazaki M. Regulation of stearoyl-CoA desaturases and role in metabolism. Prog. Lipid Res., 43, 91-104 (2004).

23) Ntambi JM, Miyazaki M. Recent insights into stearoyl-CoA desaturase-1. Curr. Opin. Lipidol., 14, 255-261 (2003).

24) Dobrzyn A, Ntambi JM. Stearoyl-CoA desaturase as a new drug target for obesity treatment. Obes. Rev., 6, 169-174 (2005).

25) Dobrzyn A, Ntambi JM. The role of stearoyl-CoA desaturase in body weight regulation. Trends Cardiovasc. Med., 14, 77-81 (2004).

26) Jakobsson A, Westerberg R, Jacobsson A. Fatty acid elongases in mammals: their regulation and roles in metabolism. Prog. Lipid Res., 45, 237-249 (2006).

27) Tamura K, Makino A, Hullin-Matsuda F, Kobayashi T, Furihata M, Chung S, Ashida S, Miki T, Fujioka T, Shuin T, Nakamura Y, Nakagawa H. Novel lipogenic enzyme ELOVL7 is involved in prostate cancer growth through saturated long-chain fatty acid metabolism. Cancer Res., 69, 8133-8140 (2009).

28) Green CD, Ozguden-Akkoc CG, Wang Y, Jump DB, Olson LK. Role of fatty acid elongases in determination of de novo synthesized monounsaturated fatty acid species. J. Lipid Res., 51, 1871-1877 (2010).

29) Marcel YL, Christiansen K, Holman RT. The preferred metabolic pathway from linoleic acid to arachidonic acid in vitro. Biochim. Biophys. Acta, 164, 25-34 (1968).

30) Brenna JT, Kothapalli KSD, Park WJ. Alternative transcripts of fatty acid desaturase (FADS) genes. Prostaglandins Leukot. Essent. Fatty Acids, 82, 281-285 (2010).

31) Unger RH. Minireview: weapons of lean body mass destruction: the role of ectopic lipids in the metabolic syndrome. Endocrinology, 144, 5159-5165 (2003).

32) Miyazaki M, Kim Y-C, Gray-Keller MP, Attie AD, Ntambi JM. The biosynthesis of hepatic cholesterol esters and triglycerides is impaired in mice with a disruption of the gene for stearoyl-CoA desaturase 1. J. Biol. Chem., 275, 30132-30138 (2000).

33) Ntambi JM, Miyazaki M, Stoehr JP, Lan H, Kendziorski CM, Yandell BS, Song Y, Cohen P, Friedman JM, Attie AD. Loss of stearoyl-CoA desaturase-1 function protects mice against adiposity. Proc. Natl. Acad. Sci. U.S.A., 99, 11482-11486 (2002).

34) Dobrzyn P, Dobrzyn A, Miyazaki M, Cohen P, Asilmaz E, Hardie DG, Friedman JM, Ntambi JM. Stearoyl-CoA desaturase 1 deficiency increases fatty acid oxidation by activating AMP-activated protein kinase in liver. Proc. Natl. Acad. Sci. U.S.A., 101, 6409-6414 (2004).

35) Rahman SM, Dobrzyn A, Dobrzyn P, Lee S-H, Miyazaki M, Ntambi JM. Stearoyl-CoA desaturase 1 deficiency elevates insulinsignaling components and down-regulates protein-tyrosine phosphatase 1B in muscle. Proc. Natl. Acad. Sci. U.S.A., 100, 11110-11115 (2003).

36) Wang D, Wei Y, Pagliassotti MJ. Saturated fatty acids promote endoplasmic reticulum stress and liver injury in rats with hepatic steatosis. Endocrinology, 147, 943-951 (2006).

37) Wei Y, Wang D, Gentile CL, Pagliassotti MJ. Reduced endoplasmic reticulum luminal calcium links saturated fatty acid-mediated endoplasmic reticulum stress and cell death in liver cells. Mol. Cell. Biochem., 331, 31-40 (2009).

38) Listenberger LL, Han X, Lewis SE, Cases S, Farese RV Jr, Ory DS, Schaffer JE. Triglyceride accumulation protects against fatty acidinduced lipotoxicity. Proc. Natl. Acad. Sci. U.S.A., 100, 3077-3082 (2003).

39) Cao H, Gerhold K, Mayers JR, Wiest MM, Watkins SM, Hotamisligil GS. Identification of a lipokine, a lipid hormone linking adipose tissue to systemic metabolism. Cell, 134, 933-944 (2008). 